\title{
Breaking the $10 \mathrm{~nm}$ barrier in hard-X-ray focusing
}

\author{
Hidekazu Mimura ${ }^{1 \star}$, Soichiro Handa ${ }^{1}$, Takashi Kimura' ${ }^{1}$ Hirokatsu Yumoto ${ }^{2}$, Daisuke Yamakawa , \\ Hikaru Yokoyama', Satoshi Matsuyama', Kouji Inagaki ${ }^{1}$, Kazuya Yamamura ${ }^{3}$, Yasuhisa Sano', \\ Kenji Tamasaku ${ }^{4}$, Yoshinori Nishino ${ }^{4}$, Makina Yabashi ${ }^{4}$, Tetsuya Ishikawa ${ }^{4}$ and Kazuto Yamauchi ${ }^{1,3}$
}

\begin{abstract}
Hard X-rays have exceptional properties that are useful in the chemical, elemental and structure analysis of matter. Although single-nanometre resolutions in various hard-X-ray analytical methods are theoretically possible with a focused hard-X-ray beam, fabrication of the focusing optics remains the main hurdle. Aberrations owing to imperfections in the optical system degrade the quality of the focused beam'. Here, we describe an in situ wavefront-correction approach to overcome this and demonstrate an X-ray beam focused in one direction to a width of $7 \mathrm{~nm}$ at $20 \mathrm{keV}$. We achieved focal spot improvement of the X-ray nanobeam produced by a laterally graded multilayer mirror ${ }^{2}$. A grazing-incidence deformable mirror $^{3}$ was used to restore the wavefront shape. Using this system, ideal focusing conditions are achievable even if hard$X$-ray focusing elements do not achieve sufficient performance. It is believed that this will ultimately lead to single-nanometre spatial resolution in X-ray analytical methods.
\end{abstract}

Synchrotron radiation facilities produce high-quality light with wavelengths ranging from the infrared to hard-X-ray regions. The use of hard $\mathrm{X}$-rays with energies higher than several kiloelectronvolts in conjunction with analysis methods such as $\mathrm{X}$-ray diffraction, $\mathrm{X}$-ray fluorescence, $\mathrm{X}$-ray absorption and $\mathrm{X}$-ray photoelectron spectroscopy offers unique advantages for the investigation of the structure, elemental distribution and chemical bonding state of advanced materials and biological samples. In these analytical methods, the resolution, signal strength and contrast must be as high as possible. In this regard, the development of a hard-X-ray focusing device is important for meeting these demands. To focus light, it is necessary to take advantage of its interactions with matter, such as diffraction, reflection and refraction. There are a variety of hard-X-ray focusing optical systems such as mirrors ${ }^{4}$, zone plates ${ }^{5}$, refractive lenses ${ }^{6}$ and multilayer Laue lenses ${ }^{7}$. The minimum achievable spot size has been theoretically investigated by many researchers ${ }^{8-10}$, and it has been concluded that sizes below $10 \mathrm{~nm}$ are feasible with kiloelectronvolt $\mathrm{X}$-rays. That is, hard-X-ray analytical techniques have the potential for single-nanometre spatial resolution.

However, in such discussions, the imperfections of the focusing elements have not been entirely considered. Rayleigh's quarterwavelength rule ${ }^{1}$ states that if the wavefront aberration exceeds a quarter of a wavelength, the quality of the retinal image will be significantly impaired. This rule is also applicable to simple light-focusing optical systems. The wavefront error of the focused beam distorts the shape of the intensity profile on the focal plane and spreads the beam. The short wavelength of X-rays demands unprecedented accuracy in the manufacturing of the optical components to form an ideal spherical wave. The main obstacle in reaching the ultimate minimum limit is the difficulty in fabricating optical elements of sufficient quality.

On the other hand, various scanning-type microscopes that use electron and ion beams, such as the scanning transmission electron microscope $e^{11}$ and the atom-probe field-ion microscope ${ }^{12}$, already have atomic-level resolution capability. When using these devices, to obtain a nanometre-sized electron or ion beam, the electric and magnetic fields of the electron lenses are finely tuned in situ, while simultaneously viewing the sample images. Furthermore, in many kinds of imaging system, from visible microscopes to space telescopes, adaptive optical methods have been implemented to alleviate the degradation owing to wavefront aberration and have contributed to realizing diffraction-limited resolution ${ }^{13}$.

Here, we describe an in situ wavefront analysis and correction technique to compensate for aberrations in hard-X-ray focusing optics $^{14-16}$ and achieve a diffraction-limited spot size of $7 \mathrm{~nm}$.

We propose a grazing-incidence total-reflection mirror with a highly controllable shape as an X-ray phase compensator. The phase shift $\Phi$ of an X-ray beam reflected from a bump on a mirror surface is given by

$$
\Phi=2 d \sin \theta / \lambda
$$

where $d$ is the height of the bump on the total reflection mirror and $\theta$ is the incident angle of the mirror. When the incident angle is about $4 \mathrm{mrad}, \lambda$ is $0.6 \AA$ and $d$ is $1.5 \mathrm{~nm}, \Phi$ is calculated to be 0.2 wave period. If the mirror surface is formed with a nanometre-level precision, the wavefront of the reflected beam can be controlled to an accuracy of 0.1 wave period.

Figure 1 shows a schematic view of the proposed optical system for in situ X-ray wavefront compensation. The effect of the figure error of the focusing mirror on the focusing properties is compensated by controlling the wavefront of the incoming X-rays with a deformable mirror upstream of the focusing mirror.

To correct errors in the waveform, it is necessary to know what these errors are. In the visible to soft-X-ray spectral range, a reference spherical wave from a minute pinhole can be easily produced and, using phase-shifting methods, the objective wavefront errors can be computed ${ }^{17}$. However, in the hard-X-ray range, it is difficult to form a reference spherical wave that can interact with the main X-ray beam. For this reason, we proposed a phase-retrieval method using the intensity profiles around the beam waist ${ }^{18}$. The wavefront on the pupil of a focusing element can be calculated, using an iterative program, from the precise intensity profiles. We have already succeeded in probing the intensity profiles

\footnotetext{
1Department of Precision Science and Technology, Graduate School of Engineering, Osaka University, 2-1 Yamada-oka, Suita, Osaka 565-0871, Japan,

${ }^{2}$ Japan Synchrotron Radiation Research Institute (JASRI)/SPring-8, 1-1-1 Kouto, Sayo-cho, Sayo-gun, Hyogo 679-5148, Japan, ${ }^{3}$ Research Center for Ultra-Precision Science and Technology, Graduate School of Engineering, Osaka University, 2-1 Yamada-oka, Suita, Osaka 565-0871, Japan,

${ }^{4}$ RIKEN/SPring-8, 1-1-1 Kouto, Sayo-cho, Sayo-gun, Hyogo 679-5148, Japan. *e-mail: mimura@prec.eng.osaka-u.ac.jp.
} 

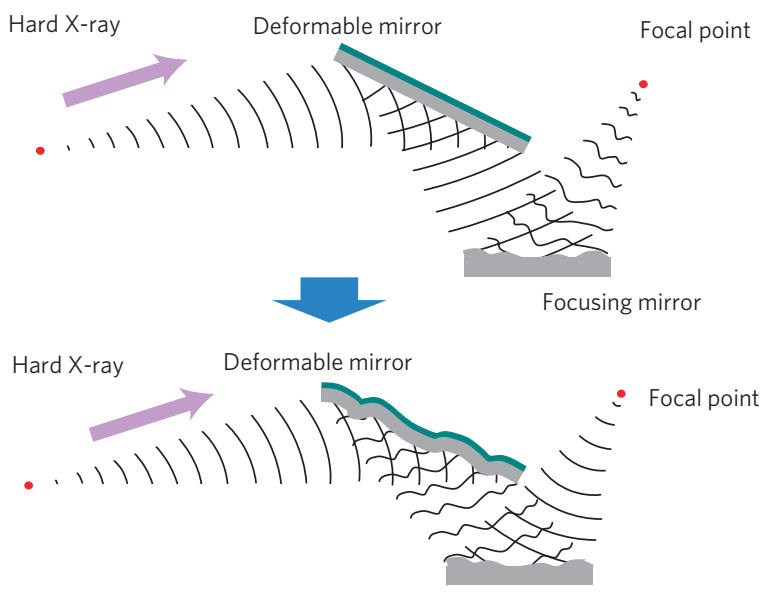

Focusing mirror

Figure 1 | Concept of an adaptive optical system for hard-X-ray focusing. In the upper panel, the surface profile of the deformable mirror is flat and the wavefront of the X-ray beam reflected on the focusing mirror is distorted owing to figure errors. The focusing state around the focal point is not ideal. In contrast, in the lower panel, the surface profile of the upstream mirror is deformed to compensate for the wavefront error. As a result, an ideally focused beam is realized.

having fourth-order satellite structures and in determining the wavefront error of an X-ray focusing mirror in situ ${ }^{19}$.

The X-ray line focusing system shown in Fig. 1 was constructed, consisting of a deformable mirror and a laterally graded multilayer focusing mirror. The average incident angle of the focusing mirror was $7 \mathrm{mrad}$. The length of the mirror was $80 \mathrm{~mm}$. The distance between the centre position of the focusing mirror and the focal point was $75 \mathrm{~mm}$. The X-ray energy used was $20 \mathrm{keV}$. The multilayer film was made up of a series of 20 platinum/carbon bilayers. Using this arrangement, the full-width at half-maximum of the designed focused beam was approximately $7 \mathrm{~nm}$ at $20 \mathrm{keV}$. The elliptically curved substrate surface of the focusing mirror was fabricated by elastic emission machining and stitching interferometry ${ }^{20-22}$. The multilayer film was coated by a magnetron sputtering method.

The system was installed in the 1-km-long beamline (BL29XUL) of SPring-8 (ref. 23). The monochromatic X-rays were first reflected by the deformable mirror, again by the multilayer mirror and finally linearly focused onto the focal plane. The incident angle and length of the deformable mirror were approximately $4 \mathrm{mrad}$ and $150 \mathrm{~mm}$, respectively. At an experimental hutch situated $1 \mathrm{~km}$ from a $50-\mu \mathrm{m}$-wide horizontal slit located behind the monochromator, almost fully coherent illumination of the mirror was possible. The surface shape was monitored and maintained by a feedback system with a Fizeau interferometer facing the deformable mirror. First, when the surface profile of the deformable mirror was set to be flat, the intensity profiles around the focal point were measured. Following this, the wavefront error profiles were recovered using a phase-retrieval method based on intensity measurements in a series of planes near the focal plane. After adjusting the surface profile of the deformable mirror to the profile to compensate the wavefront error, the focused beam profiles were evaluated again. Figure $2 \mathrm{a}$ shows the surface profile input into the controller of the deformable mirror and that measured by the Fizeau interferometer. Figure $2 \mathrm{~b}$ shows the intensity profiles before and after wavefront correction at the point where the minimum focused beam size was measured before wavefront correction. As shown clearly, the shape of the intensity profile is further improved, resulting in the breaking of the $10 \mathrm{~nm}$ barrier in hard-X-ray focusing. In this study, it is crucial to be capable of directly measuring intensity profiles with a resolution of less than $10 \mathrm{~nm}$. As a

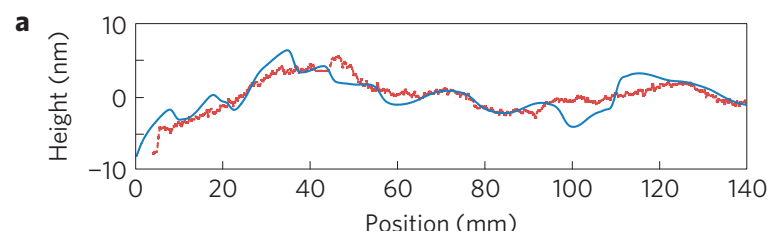

b

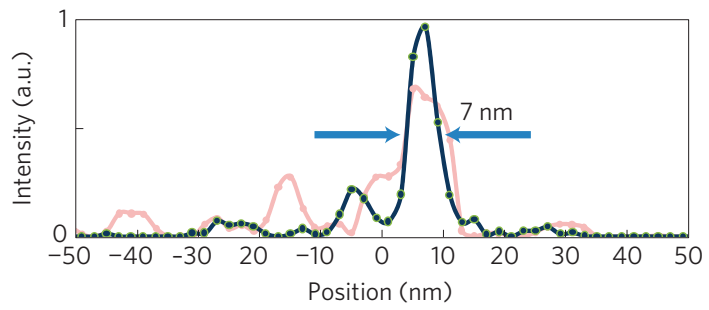

Figure 2 | Improvement in the intensity profiles on focal planes. a, Profiles of the deformable mirror. The blue profile is input into the controller of the deformable mirror and the red one is measured by a Fizeau interferometer. The Fizeau interferometer having a flat reference surface is placed facing the deformable mirror to monitor the surface profile. The method used to deform and maintain the mirror shape is explained in the Methods section. b. Comparison between the intensity profiles of the focused beam measured after (black line) and before (pink line) wavefront correction. The method used to measure the intensity distribution is described in the Methods section.

result of improvements in thermal and vibration control owing to the methods mentioned in the Methods section, the best focusing state with a sub-10-nm beam size could be maintained for at least half a day.

Figure $3 a-d$ shows a comparison between the experimentally measured profiles and the profiles recovered during the phaseretrieval calculations. The intensity profiles shown in Fig. 3a,b were measured in the focal plane, where the minimum focal size was obtained; whereas the profiles shown in Fig. $3 c$,d were measured $12 \mu \mathrm{m}$ downstream. The iterative algorithm developed as an optical metrology tool ${ }^{24}$ was applied to this study. The red profiles in Fig. $3 \mathrm{a}-\mathrm{d}$ are the recovered intensity profiles from the phaseretrieval calculations and agree well with the experimental results, indicating the reliability of the phase-retrieval calculation method. Figure $3 \mathrm{e}, \mathrm{f}$ illustrates the intensity profiles of the beam waist structure before and after wavefront correction, as predicted by the simulations. As can be seen, the distorted beam-waist structure was successfully corrected. The difference between these two sets of experimental data is only the shape of the deformable mirror.

As the peak-to-valley height of the surface profile of the deformable mirror is $10 \mathrm{~nm}$, as shown in Fig. 2a, the degree of wavefront distortion was assumed to 1.3 wave period, following equation (1). This value does not satisfy the Rayleigh quarterwavelength rule. The wavefront errors are due to the following three factors. The first is the figure error of the substrate of the focusing mirror. In this case, the surface profile corresponds to an aspheric surface with a strongly curved shape, which is difficult to measure with absolute accuracy at the nanometre scale. The second is the thickness distribution error of the multilayer film. At present, there are no methods for confirming the quality of the multilayer at the level required for diffraction-limited focusing. Even if a multilayer mirror has high reflectivity, unwanted phase shift might occur on reflection. An at-wavelength focusing test is the only diagnostic technique for phase-shift distortion. The third factor is misalignment when positioning the focusing mirror. For example, the focal length and incident angle strongly affect the focusing state, and should be adjusted to within a few micrometres and $1 \times 10^{-7} \mathrm{rad}$, respectively. For these reasons, development of a system capable of wavefront measurement and correction is 


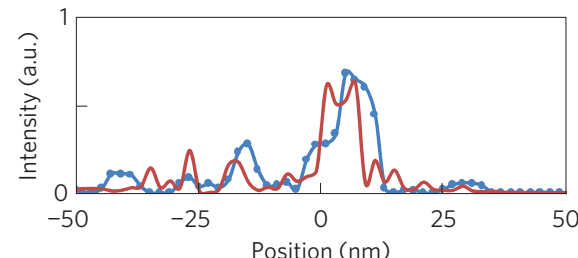

c
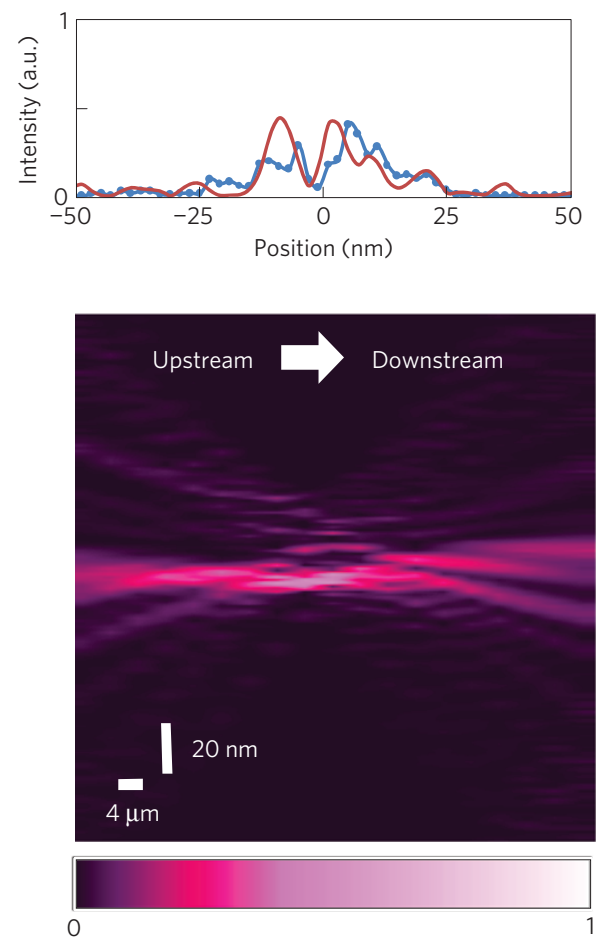

Intensity (a.u.) b

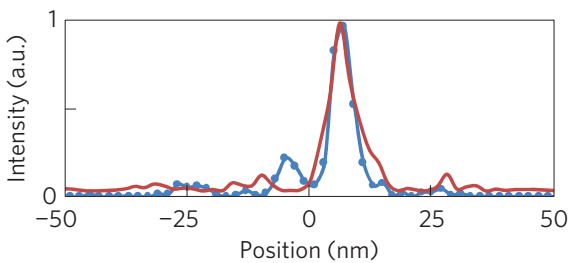

d

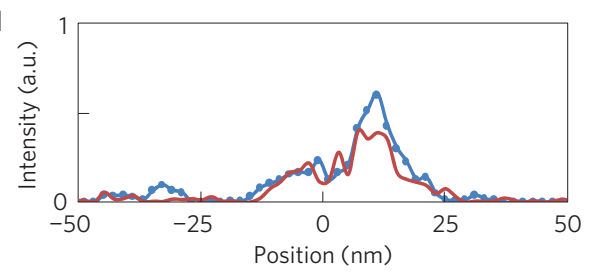

f
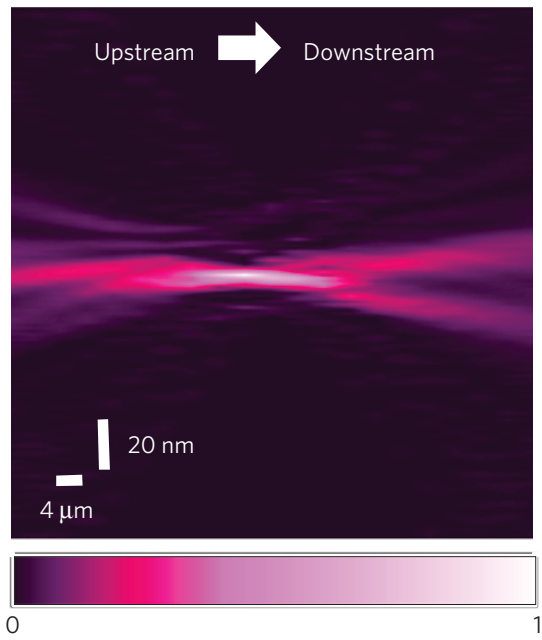

Intensity (a.u.)

Figure 3 | Comparison between the measured profile and recovered intensity profile of the focused beam in the phase-retrieval calculations.

$\mathbf{a}, \mathbf{b}$, Intensity profiles before (a) and after (b) wavefront correction on the plane, where the minimum focused beam profile is obtained before wavefront correction. c,d, Intensity profiles before (c) and after (d) wavefront correction, $12 \mu \mathrm{m}$ downstream from the points corresponding to $\mathbf{a}$ and $\mathbf{b}$. The blue curves in $\mathbf{a}-\mathbf{d}$ are the experimentally measured profiles. The red profiles are recovered by phase-retrieval calculations. e,f, Predicted beam-waist structure before (e) and after (f) wavefront correction. The X-ray energy is $20 \mathrm{keV}$.

considered to be the most realistic approach towards achieving diffraction-limited X-ray focusing.

The short wavelength of hard X-rays means that they can potentially be focused to within a nanometre area. A wavefront analysis and correction system that could achieve a degree of controllability of 0.1 wave period could support any X-ray focusing system and allow a focused beam size of $1 \mathrm{~nm}$. Such narrow beams will produce intensive X-rays and stimulate the advancement of nonlinear X-ray optics fields ${ }^{25}$. Furthermore, X-ray free-electron lasers with single nanometre-scale beams ${ }^{26}$ can be expected to produce a highly localized electromagnetic field strong enough to overcome the Schwinger limit ${ }^{27}$. Thus, such a focusing system will not only lead to breakthroughs in X-ray analytic techniques, but will also open new frontiers in X-ray physics.

\section{Methods}

Laterally graded multilayer mirror for hard-X-ray focusing. The laterally graded multilayer mirror allows high-performance hard-X-ray focusing with very low chromatic aberration. The monochromaticity of the incident X-rays has no effect on the size of the focused beam, and a beam size of less than $1 \mathrm{~nm}$ is possible ${ }^{10}$. The mirror substrate was fabricated by computer-controlled figuring using accurately measured surface profile data. The main machining method was elastic-emission machining with a removal depth controllability of $0.1 \mathrm{~nm}$ and a spatial resolution of $0.3 \mathrm{~mm}$ in figuring $\mathrm{g}^{20}$. Metrology consisting of microstitching interferometry and relative-angle determinable stitching interferometry was specially developed for measuring the surface profiles of the X-ray focusing mirror ${ }^{21,22}$. A laterally graded multilayer coating was applied using a magnetron sputtering method (Ulvac.). A small aperture positioned between the mirror and cathodes was used to limit the deposition area. A graded-thickness film was deposited by scanning the mirror stage at various speeds. Twenty platinum/carbon bilayers were coated on the mirror substrate. Further details of the design of the laterally graded multilayer mirror are described in the Supplementary Information.

Deformable mirror. The substrate of the deformable mirror was a silicon block with a length of $150 \mathrm{~mm}$, a width of $50 \mathrm{~mm}$ and a thickness of $10 \mathrm{~mm}$. On the back face of the substrate, 18 lines of piezoelectric elements were attached by gluing at high temperature. After that, the substrate was smoothed and figured flat by elastic-emission machining. As the difference in the coefficients of thermal expansion between the piezoelectric elements and the silicon substrate caused unwanted bending of the substrate, the temperature of the experimental room was kept constant to within $0.2^{\circ} \mathrm{C}$. The surface profile was measured with a Fizeau interferometer at $3 \mathrm{~min}$ intervals. Each voltage-supplying piezoelectric element was retuned individually so that the surface profile of the deformable mirror was tuned to the profiles input to the computer. The voltages were determined on the basis of the Bernoulli-Euler beam theory. Further details of the optical system are described in the Supplementary Information.

Beam intensity profile measurement. As explained in ref. 19, a phase object with the shape of a microbridge structure was inserted into the beam waist. The difference between the microbridge structure used in this study and in ref. 19 is its width, because the focal depth of the beam waist is shorter than that in ref. 19. The platinum microbridge structure with a width of $1 \mu \mathrm{m}$ was fabricated using a focused-ion-beam method. The surface roughness on the bridge was 
$1.43 \mathrm{~nm}$ (r.m.s.) over a 500-nm-square area, which was directly confirmed by atomic force microscopy. A phase shift occurred at the boundary between the $\mathrm{X}$-rays propagating inside and outside the phase object. The intensity of the X-rays diffracted from the boundary was proportional to that of the illumination beam at the boundary. The intensity profile of the focused beam could be measured by detecting the diffracted $\mathrm{X}$-rays at the dark-field position while inserting the phase object into the beam waist. An avalanche photodiode detector was used to measure the diffracted X-rays. Further details of the above procedure are described in ref. 19. The temperature in the experimental hutch was controlled to be $29 \pm 0.1^{\circ} \mathrm{C}$ to prevent any unwanted drift. To guard against vibrations, all equipment, including the CCD (charge-coupled device) camera, that produced sound and vibrations in the hutch was completely stopped during measurements.

Phase-retrieval calculation. A new phase-retrieval algorithm was developed to determine the wavefront error distributions of focusing optics, in which many intensity profiles for different planes near the focal plane are considered ${ }^{24}$. The iterative algorithm is based on the nonlinear optimization ${ }^{28}$ and the angular spectrum methods. In this letter, 18 intensity profiles around the focal point were input into the simulation code. After more than 2,500 iterations, the wavefield distributions at the pupil became stationary so that the sum-of-squared errors between the recovered profiles and the measured profiles were minimized. Further details of the phase-retrieval process are described in the Supplementary Information.

Received 2 July 2009; accepted 22 October 2009; published online 22 November 2009; corrected online 2 December 2009

\section{References}

1. Born, M. \& Wolf, E. Principles of Optics Seventh edition 527-532 (Cambridge Univ. Press, 1999).

2. Morawe, C., Pecci, P., Peffern, J. C. \& Ziegler, E. Design and performance of graded multilayers as focusing elements for X-ray optics. Rev. Sci. Instrum. 70, 3227-3232 (1999).

3. Signorato, R., Hignette, O. \& Goulon, J. Multi-segmented piezoelectric mirrors as active/adaptive optics components. J. Synchrotron. Rad. 5, 797-800 (1998)

4. Mimura, H. et al. Efficient focusing of hard-X-rays to $25 \mathrm{~nm}$ by a total reflection mirror. Appl. Phys. Lett. 90, 051903 (2007).

5. Suzuki, Y., Takeuchi, A., Takano, H. \& Takenaka, H. Performance test of Fresnel zone plate with $50 \mathrm{~nm}$ outermost zone width in hard $\mathrm{X}$-ray region. Jpn. J. Appl. Phys. 44, 1994-1998 (2005).

6. Schroer, C. G. et al. Hard X-ray nanoprobe based on refractive X-ray lenses. Appl. Phys. Lett 87, 124103 (2005).

7. Kang, H. C. et al. Nanometer linear focusing of hard-X-rays by a multilayer Lue lens. Phy. Rev. Lett. 96, 127401 (2006).

8. Bergemann, C., Keymeulen, H. \& van der Veen, J. F. Focusing X-ray beams to nanometer dimensions. Phy. Rev. Lett. 91, 204801 (2003).

9. Schroer, C. G. \& Lengeler, B. Focusing hard-X-rays to nanometer dimensions by adiabatically focusing lenses. Phy. Rev. Lett. 94, 054802 (2005).

10. Morawe, Ch., Guigay, J.-P., Mocella, V. \& Ferrero, C. An analytical approach to estimating aberrations in curved multilayer optics for hard X-rays: 2 interpretation and application to focusing experiments. Opt. Exp. 16, 16138-16150 (2008).

11. Varela, M. et al. Atomic-resolution imaging of oxidation states in manganites. Phy. Rev. B 79, 085117 (2009).

12. Kelly, T. F. \& Miller, M. K. Atom probe tomography. Rev. Sci. Instrum. 78, 031101 (2007).
13. Albert, O., Sherman, L., Mourou, G., Norris, T. B. \& Vdovin, G. Smart microscope: An adaptive optics learning system for aberration correction in multiphoton confocal microscopy. Opt. Lett. 25, 52-54 (2000).

14. Hignette, O., Cloetens, P., Rostaing, G., Bernard, P. \& Morawe, C. Efficient sub $100 \mathrm{~nm}$ focusing of hard-X-rays. Rev. Sci. Instrum. 76, 063709 (2005).

15. Signorato, R., Häusermann, D., Somayazulu, M. \& Carré, J.-F. Performance of an adaptive $\mu$-focusing Kirkpatrick-Baez system for high pressure studies at the Advanced Photon Source. Proc. SPIE-Int. Soc. Opt. Eng. 5193, 112-123 (2004).

16. Kimura, T. et al. Wavefront control system for phase compensation in hard-X-ray optics. Jpn. J. Appl. Phys. 48, 072503 (2009).

17. Smartt, R. N. \& Steel, W. H. Theory and application of point-diffraction interferometers. Jpn. J. Appl. Phys. 14, 351-356 (1975).

18. Yumoto, H. et al. At-wavelength figure metrology of hard-X-ray focusing mirrors. Rev. Sci. Instrum. 77, 063712 (2006).

19. Mimura, H. et al. Direct determination of the wave field of an X-ray nanobeam. Phy. Rev. A 77, 015812 (2008).

20. Yamauchi, K., Mimura, H., Inagaki, K. \& Mori, Y. Figuring with subnanometre-level accuracy by numerically controlled elastic emission machining. Rev. Sci. Instrum. 73, 4028-4033 (2002).

21. Yamauchi, K. et al. Microstitching interferometry for X-ray reflective optics. Rev. Sci. Instrum. 74, 2894-2898 (2003).

22. Mimura, H. et al. Relative angle determinable stitching interferometry for hard-X-ray reflective optics. Rev. Sci. Instrum. 76, 045102 (2005).

23. Tamasaku, K. et al. SPring-8 RIKEN beamline III for coherent X-ray optics. Nucl. Instrum. Methods 467-468, 686-689 (2001).

24. Brady, G. R \& Fienup, J. R. Nonlinear optimization algorithm for retrieving the full complex pupil function. Opt. Exp. 14, 474-486 (2006).

25. Tamasaku, K. \& Ishikawa, T. Interference between Compton scattering and X-ray parametric down-conversion. Phys. Rev. Lett. 98, 244801 (2007).

26. Mimura, H. et al. Focusing mirror for X-ray free-electron lasers. Rev. Sci. Instrum. 79, 083104 (2008).

27. Schwinger, J. On gauge invariance and vacuum polarization. Phys. Rev. B 82, 664-679 (1951).

28. Fienup, J. R. Phase retrieval algorithms-a comparison. Appl. Opt. 21, 2758-2769 (1982)

\section{Acknowledgements}

We thank G. Brady for the use of the advanced phase retrieval algorithm. The use of the 1-km-long beamline (BL29XUL SPring-8) was supported by RIKEN. This research was supported by a Grant-in-Aid for Specially Promoted Research 18002009, 2009, the Global COE Program, Center of Excellence for Atomically Controlled Fabrication Technology, 2009, and the X-ray Free Electron Laser Utilization Research Project, 2009, from the Japanese Ministry of Education, Culture, Sports, Science and Technology.

\section{Author contributions}

This experiment was carried out mainly by H.M., S.H., T.K., H. Yumoto, D.Y. and H. Yokoyama. The multilayer mirror and deformable mirror were fabricated by S.M., K.I., Y.S. and K. Yamamura. The conditions for this experiment were set up by K.T., Y.N. and M.Y. Experimental planning was carried out by H.M., T.I. and K. Yamauchi.

\section{Additional information}

Supplementary information accompanies this paper on www.nature.com/naturephysics. Reprints and permissions information is available online at http://npg.nature.com/ reprintsandpermissions. Correspondence and requests for materials should be addressed to H.M. 